\title{
HIGH PRESSURE OPTICAL MEASUREMENTS OF SIZES, VELOCITIES AND LONGITUDINAL POSITIONS OF BUBBLES
}

\author{
H. MENG, P. BOOT and C. W. M. VAN DER GELD \\ Faculty of Mechanical Engineering, Eindhoven University of Technology, P.O. Box 513, 5600 MB \\ Eindhoven, The Netherlands
}

(Received 9 January 1994; in revised form 14 June 1994)

\begin{abstract}
This paper presents an optical system for taking shadow photographs of bubbles in a high-pressure evaporator tube. With this system and interpretation principles, not only the size and velocity of a bubble can be calculated from its shadow images on photos, but also its longitudinal position on the optical axis may be estimated from the size of a "bright spot" in its shadow image. A simple relation based on geometrical optics is applied. Performance of the new system and validity of the interpretation principles were examined through three series of calibration tests. Relative deviations of estimations to actual values of sizes and longitudinal positions of bubbles are within 1 and $5 \%$ respectively. Application at high-pressure was successful. Due to the general nature of the system and the interpretation principles, results may be extended to more dispersed two-phase flow situations.
\end{abstract}

Key Words: photographic, high-pressure tube, bubble

\section{INTRODUCTION}

Investigations of hydrodynamics and transport mechanisms in dispersed two-phase flow systems have been conducted with a variety of experimental techniques (e.g. Hewitt 1978; Jones 1983; Cheremisinoff 1986; and so on). Studies often quantify size, velocity and distribution of the discrete phase. The complexity and variety of such two-phase systems requires not only that careful considerations be given to ensure high quality data but also that specific arrangements be made for each individual situation. In this paper attention is focused on bubbly flows and optical measurements under high pressure conditions.

A measuring technique applicable to a bubbly flow in a high-pressure tube should resist high temperature and highly corrosive two-phase mixtures meanwhile should cause little disturbance to the flow. Among the many kinds of measuring techniques utilized in the Laboratory of Multiphase Flows and Heat Transfer in Eindhoven University of Technology (Van der Geld 1985; Meng 1993), an optical system was developed to visualize local parameters on bubbles. With a pair of specially coated windows and a careful analysing technique, information of sizes, velocities and longitudinal positions (on the optical axis) of bubbles in a high-pressure tube is obtained. This method, which required the mixture not to be too dense, is one of the two non-intrusive techniques utilized in our laboratory. Another is the gamma-ray densitometer method which yields time-averaged void distributions and requires a high power source for a thick stainless-steel high-pressure tube and an advanced data reduction method (Meng et al. 1992).

\section{EXPERIMENTAL APPARATUS}

Schemes of the optical system and the related window construction on the high-pressure tube are shown in figures 1 and 2 respectively.

The optical system encompasses a camera unit and an illumination unit, mounted on an optical table. The illumination unit produces a beam of parallel light by placing a point light source at the focus point of a positive lens $(f 30 \mathrm{~mm})$. The light source may be either a usual continuous light source or a flash light source which produces light pulses at an installed frequency (a "nanolight" or a stroboscope). Through windows on two sides of the test section, the parallel light 

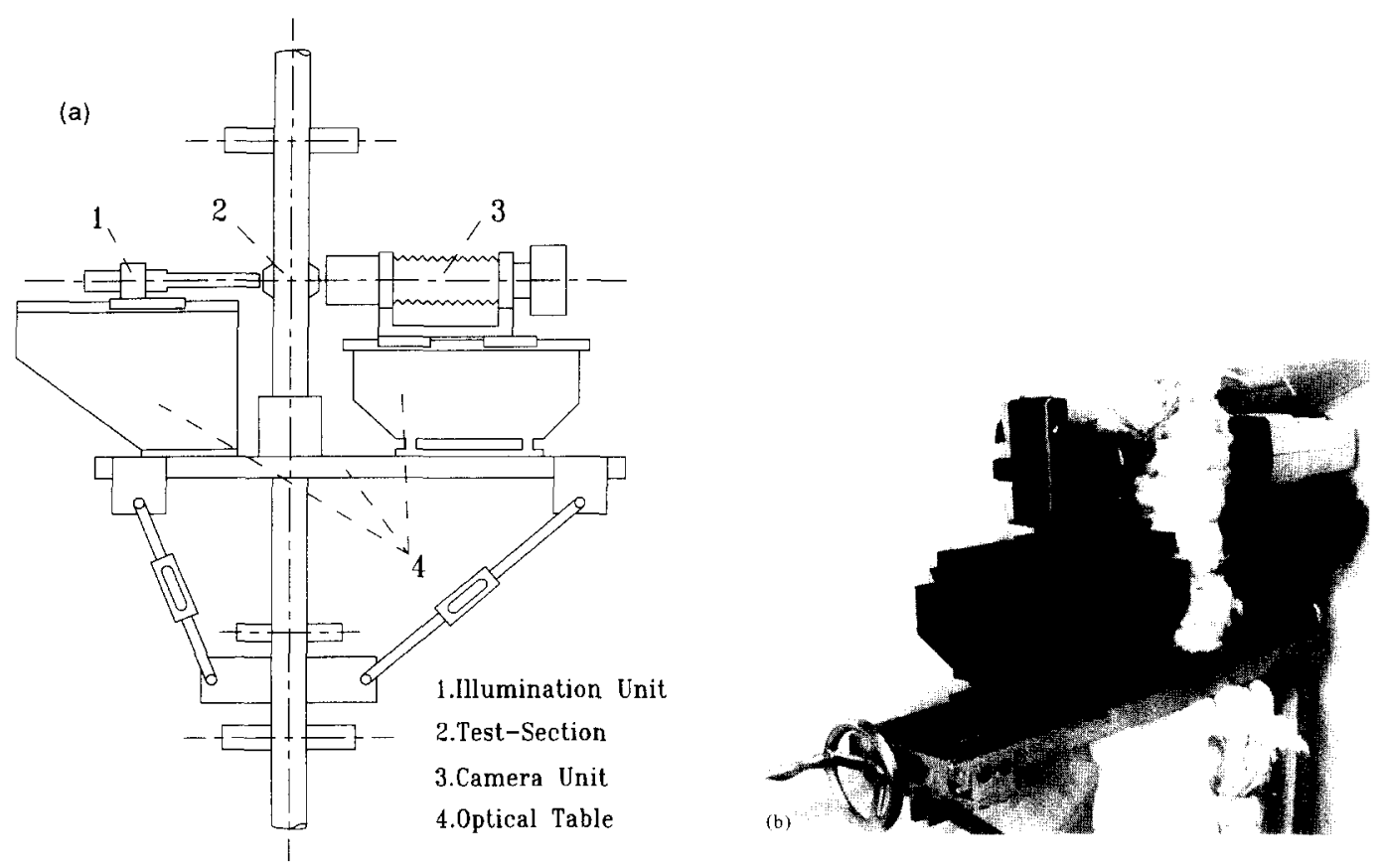

Figure 1. Schematic of the optical system.

rays pass the two-phase flow region and then are received by the camera unit. Through a camera lens (or an object lens $[f 35 \mathrm{~mm}]$ ), shadow images of bubbles are formed on the film in a camera body (PRACTICA MTL-180). The image distance, which is the distance from the object lens to the film, may be adjusted through a bellow extension mounted on an optical rail.

The window construction was designed to provide a wide-angle view as big as possible while sustaining a pressure in the tube as high as 250 bar. To be resistant to the high corrosion surroundings, both windows on two sides of the test-section are made of sapphire coated with a thin $\mathrm{Si}_{3} \mathrm{~N}_{4}$ film through a careful procedure (Van der Geld 1983).

\section{DETERMINATION OF SIZE, LONGITUDINAL POSITION AND VELOCITY OF BUBBLES}

Among various photographic methods the shadow-photograph method is the simplest optical manifestation of density changes (Hewitt 1978). When a parallel beam of light passes through

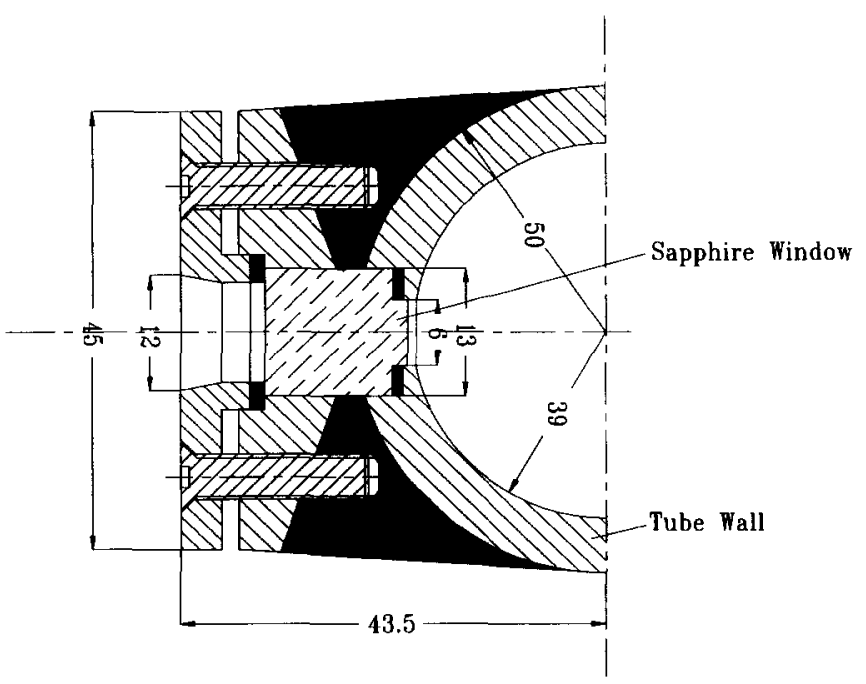

Figure 2. Schematic of the window construction. 
bubble-water region in a test-section, deflection of adjacent rays will differ because of refractive index variations. The parallel light rays will converge or diverge, causing illumination to be more or less intense.

The most accurate way to analyze image formation is to trace paths of light rays through the entire system (Klein 1986). This is now done for a spherical bubble floating in the water, with reference to the optical diagram of figure 3 .

When a beam of parallel light passes a spherical bubble in the water, the bubble can naturally be divided into two parts, namely the edge part and the central part, according to their effects on the parallel beam.

The edge part of the bubble diverges parallel light rays due to its curvature, resulting in a shadow-contour on the film. From the diameter of this shadow-contour, $D_{\mathrm{s}}$, the diameter of the bubble, $D_{0}$, is computed from the following equation

$$
D_{0}=D_{\mathrm{s}} \frac{F_{1}}{L_{\mathrm{lf}}-F_{1}}
$$

where $F_{1}$ is the focal length of the object lens and $L_{\mathrm{If}}$ is the distance from the film to the object lens.

The central part, on the other hand, converges parallel light rays in the present optical arrangement, resulting in a "bright spot" in the middle of the shadow-contour on the film. In terms of geometrical optics, this part of the bubble actually can be considered as an optical lens. From the film, light rays emerge as if they came from a point light source (we name it the "virtual light point") placed on the focal point of this "virtual lens". The focal length, $F_{0}$, of this "virtual lens" can approximately be calculated by [2] below (Morton 1984)

$$
F_{0}=\frac{D_{0} n_{\mathrm{d}}}{4\left(n_{\mathrm{d}}-1\right)}
$$

where $n_{\mathrm{d}}$ is the ratio of refraction indexes of this virtual lens to the ambient media. For an air bubble in water, $n_{\mathrm{d}}=1 / 1.33$ and $F_{0}$ equals to $-0.76 D_{0}$.

If we take the distance between the bubble and the object lens on the optical axis $\left(L_{\mathrm{lo}}\right)$ as the longitudinal position of the bubble, a "virtual light point" will appear at a distance $U_{\mathrm{b}}=L_{\mathrm{lo}}-F_{0}$. A light cone seems to be emitted from the "virtual light point" and is bordered by the lens rim. It appears as a "bright spot" on the film. The diameter $D_{\mathrm{b}}$ of the "bright spot" may be computed by

$$
D_{\mathrm{b}}=\left|L_{\mathrm{lf}}-V_{\mathrm{b}}\right| \frac{D_{1}}{V_{\mathrm{b}}}
$$

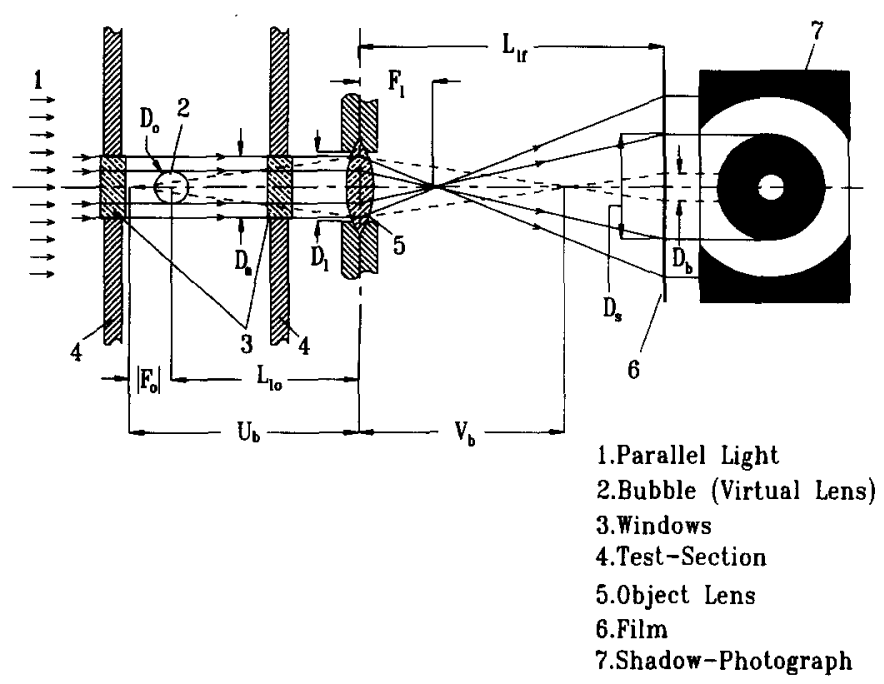

Figure 3. Image formation of a parallel light beam passing a transparent object. 
where $D_{1}$ is the diameter of the lens rim and $V_{\mathrm{b}}$ is the image distance of the "virtual light point" given by the conjugation law in geometrical optics $\left(1 / U_{\mathrm{b}}+1 / V_{\mathrm{b}}=1 / F_{1}\right)$.

Combining the above equations, in which $n_{\mathrm{d}}, D_{1}, F_{1}$, and $L_{1 \mathrm{f}}$ are known system parameters; and $D_{\mathrm{s}}$ and $D_{\mathrm{b}}$ are direct measurements from the film, we can calculate the longitudinal position of the bubble, $L_{\mathrm{lo}}$, from the following equation:

$$
L_{\mathrm{lo}}=\frac{F_{1} \frac{L_{1 \S} D_{1}}{D_{1} \pm D_{\mathrm{b}}}}{\frac{L_{1 \mathrm{f}} D_{1}}{D_{1} \pm D_{\mathrm{b}}}-F_{1}}+\frac{n_{\mathrm{d}} D_{\mathrm{s}} F_{\mathrm{l}}}{4\left(n_{\mathrm{d}}-1\right)\left(L_{1 \mathrm{f}}-F_{1}\right)}
$$

where the sign " \pm " is induced from the term $\left|L_{\mathrm{lf}}-V_{\mathrm{b}}\right|$ in [3] ("+" if $L_{\mathrm{lf}}>V_{\mathrm{b}}$; " - " if $L_{\mathrm{lf}}<V_{\mathrm{b}}$ ). Based on the Inverse Square Law of Illumination (Morton 1984), the sign of the term $\left|L_{1 \mathrm{f}}-V_{\mathrm{b}}\right|$ can be judged directly from photographs according to the lightness of bright spots relative to the lightness of the "bright background", whose size depends on the size of the minimum aperture stop, $D_{\mathrm{a}}$, as shown in figure 3 . If the bright spot is lighter or brighter than the bright background, $L_{\mathrm{lf}}<V_{\mathrm{b}}$. Otherwise $L_{\mathrm{l}}>V_{\mathrm{b}}$. In this way, the longitudinal position of a bubble in the water is established.

In addition to the measurements indicated above, the instantaneous velocity of the bubble $\left(\mathrm{VEL}_{\mathrm{b}}\right.$ ) may be deducted by using a flashing light source (a "nanolight" unit or a stroboscope). Both flashing light sources can provide a train of light pulses at an installed frequency $\left(F E Q_{n}\right)$. By coordinating the light flashing frequency and the camera diaphragm opening time, a number of image pulses are registered on one single photograph. From the distance the bubble has travelled between two light pulses, the bubble instantaneous velocity $\left(\mathrm{VEL}_{b}\right)$ can be calculated by the following equation,

$$
\mathrm{VEL}_{\mathrm{b}}=\mathrm{FEQ}_{\mathrm{n}} L_{2 \mathrm{p}}
$$

where $L_{2 \mathrm{p}}$ is the distance between two images of the same bubble measured directly from the photo.

\section{CALIBRATION TESTS}

For the validation of the new system and the interpretation principles, three series of experiments were performed using glass spheres and air bubbles.

The calibration test-setup, which simulates situations in the high-pressure tube, is shown schematically in figure 4 . The test-section is a rectangular glass container wrapped almost entirely with black tapes, except two opening windows on two sides. A continuous light source (i.e. an incandescent-filament lamp) was employed for the first and the second series and a nanolight unit or a stroboscope for the third.

In the first series of experiments, glass spheres were used as transparent objects. The glass spheres are 2 or $3 \mathrm{~mm}$ in diameter and have a refraction index of 1.51. Glass spheres therefore act as positive lenses whereas bubbles act as negative ones. Both obey the same rule of the image formation given in the previous section.

To check [1], with which the object size $\left(D_{0}\right)$ is computed from the measurement of its shadow size $\left(D_{\mathrm{s}}\right)$ on the photo (where $D_{\mathrm{s}}$ is independent of the longitudinal position of the object), a photo of three glass spheres (one is $2 \mathrm{~mm}$ and two are $3 \mathrm{~mm}$ ) was used as shown in figure 5. Comparison between computations ( $D_{0}^{\prime}$, calculated from measurement $D_{\mathrm{s}}$ ) and actual $D_{0}$, as listed in table 1, yields very satisfactory agreement.

Equation [4] is validated through a series of photos, taken with fixed distance $\left(L_{11}\right)$ between the object lens and the film. The camera unit was shifted step by step along the leading rail. Longitudinal positions of the object $\left(L_{\mathrm{lo}}^{\prime}\right)$ were calculated with [4] from measurements of shadow-contour sizes $\left(D_{\mathrm{s}}\right)$ and bright spot sizes $\left(D_{\mathrm{b}}\right)$. The series of photos and the comparison between actual and computed longitudinal positions $\left(L_{\mathrm{lo}}\right.$ and $\left.L_{\mathrm{lo}}^{\prime}\right)$ are shown in figure 6 and table 2 , respectively.

In the second series of experiments, a bubble injector was employed. Since a free rising bubble does not move in a straight line, the longitudinal position of a bubble could not be kept all the way to be the same as it left the injector. Accordingly photos had to be taken in the neighbourhood 

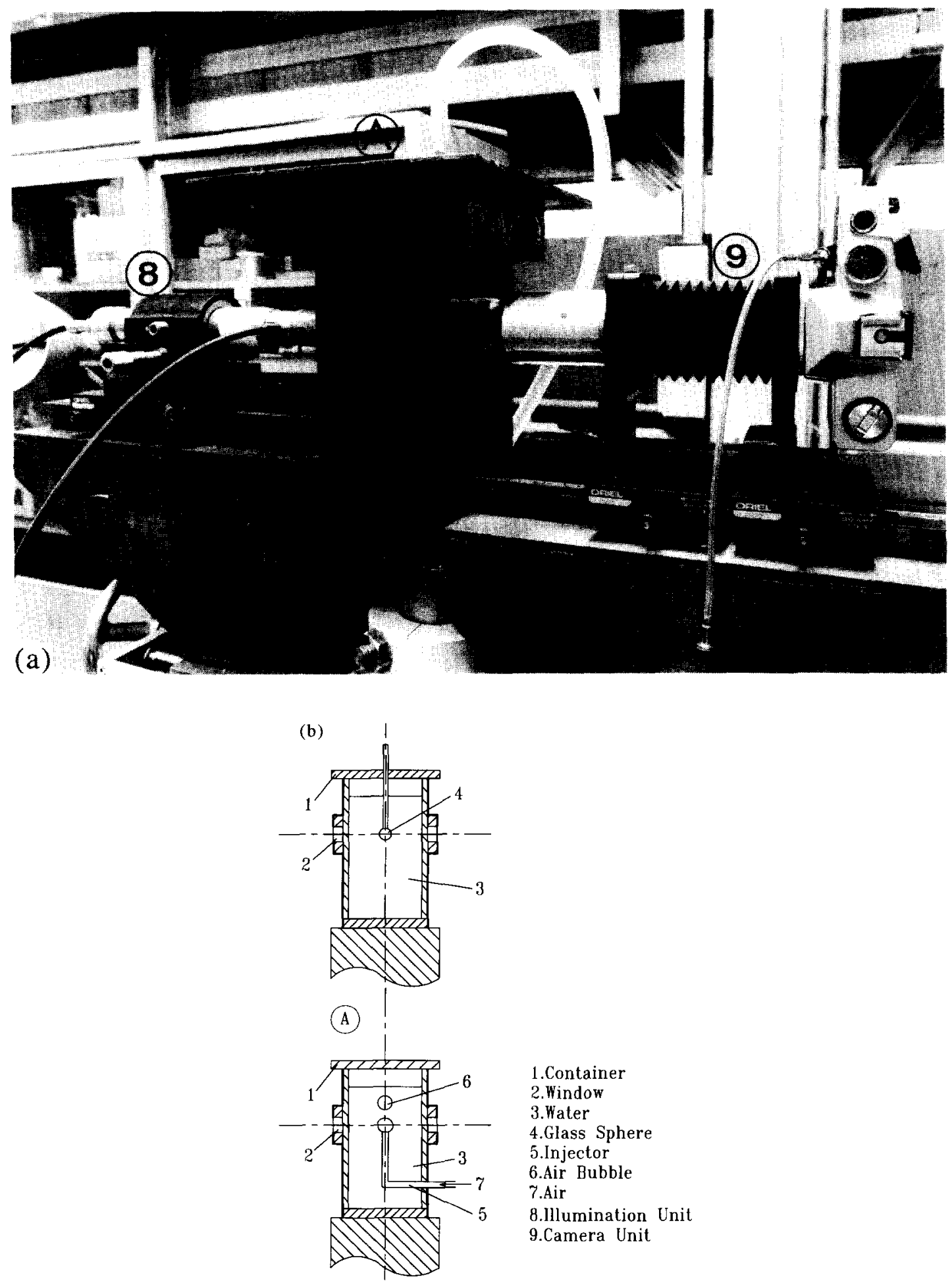

Figure 4. Diagram of the calibration test setup.

of the mouth piece of the injector where bubbles were not spherical. Measurements of sizes of shadow contours $\left(D_{\mathrm{s}}\right)$ and bright spots $\left(D_{\mathrm{b}}\right)$ were taken on one of the normal cross-sections of the bubble image on the photo with the assumption that such a cross-section has a circular shape. Longitudinal positions of bubbles $\left(L_{\mathrm{lo}}^{\prime}\right)$ were again calculated by [4] and then were compared with actual ones $\left(L_{\mathrm{lo}}\right)$, taken to be identical to the position of the mouth piece of 


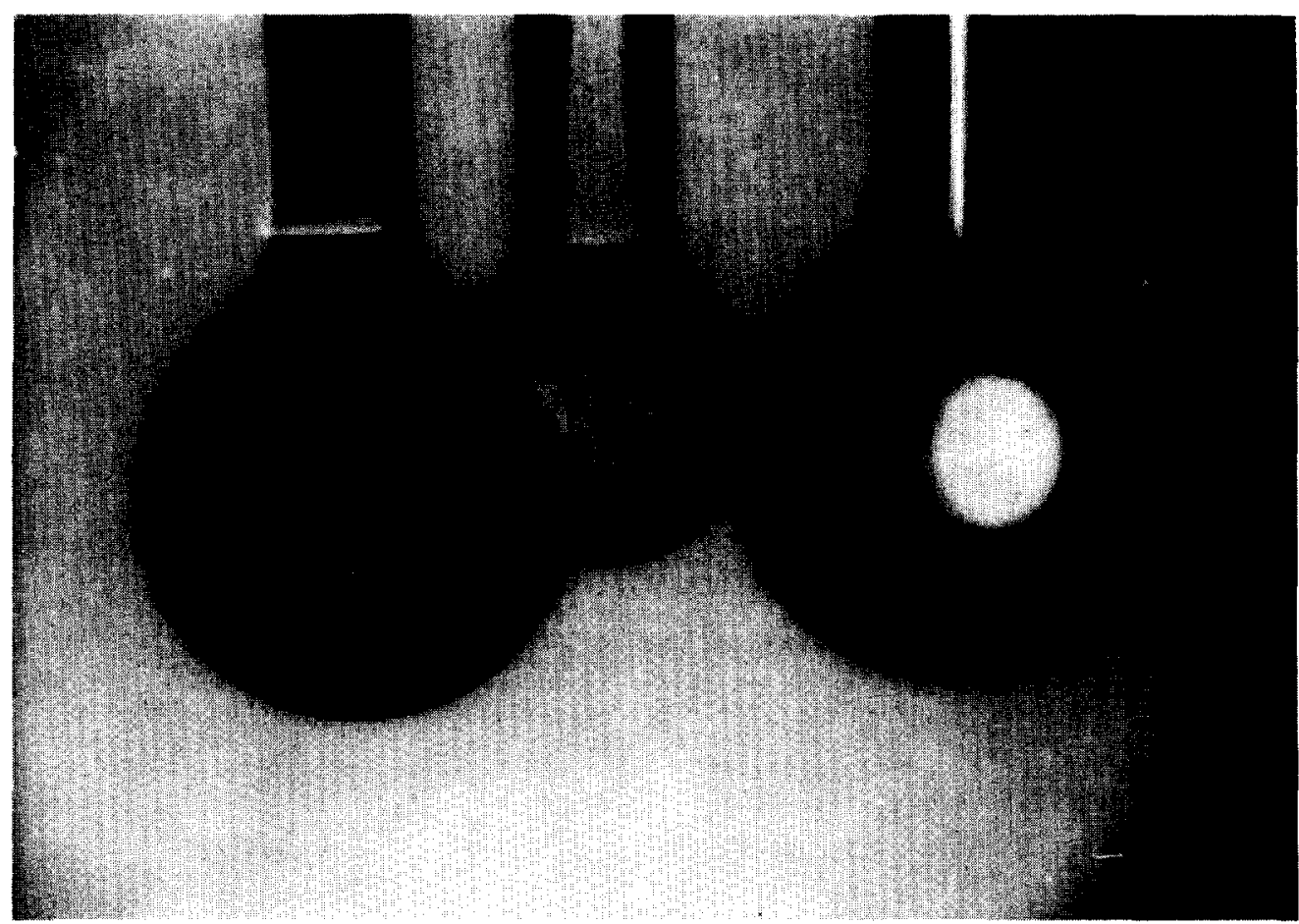

Figure 5. A photograph of glass spheres $\left(D_{0}=2 \mathrm{~mm}\right.$ or $\left.D_{0}=3 \mathrm{~mm}\right)$ in a calibration test.

the bubble-injector. Examples of photos and a list of comparisons are shown in figure 7 and table 3.

In the third series of experiments, two or three flashes from the nanolight were registered on one photo during one opening of the camera diaphragm with a certain combination of the camera shutter time and the nanolight frequency. Accordingly two or three images of some passing bubbles were recorded on one photograph. From [5], velocities of bubbles thus were computed. Two examples are shown in figure 8.

All three series of experiments have shown very satisfactory results. Relative deviations of computations from actual bubble sizes are less than $1.0 \%$, as shown in table 1 . Relative deviations of computations to actual bubble longitudinal positions are all within $5.0 \%$, as shown in tables 2 and 3 . Bubble velocities obtained are very accurate, too.

The photographic system is meant for applications in a high-pressure tube. At high pressure (e.g. 70 bar), bubbles are much smaller than under atmospheric pressure. Therefore the shape of the bubbles will be closer to spherical. This is in favour of the measuring strategy.

\section{SIZES OF VAPOUR BUBBLES IN A HIGH-PRESSURE TUBE}

There is not much information (neither experimental nor theoretical) on vapour bubbles at high pressures in a forced convection evaporator loop. Available data mostly cover pool boiling (up to 10 bar) or the departure condition of bubbles from a heated plate (for reviews, see Van Stralen 1979; Hsu \& Graham 1976).

Table 1. Comparison of computations with actual object sizes (glass spheres)

\begin{tabular}{lcccc}
\hline Index & $D_{\mathrm{s}}(\mathrm{mm})$ & $D_{0}(\mathrm{~mm})$ & $D_{0}^{\prime}(\mathrm{mm})$ & $\begin{array}{c}\left|\Delta D_{0}\right| D_{0} \\
(\%)\end{array}$ \\
\hline$(1)$ & $6.6 \pm 0.1$ & 2.00 & 1.99 & 0.5 \\
$(2)$ & $10.0 \pm 0.1$ & 3.00 & 3.02 & 0.7 \\
\hline
\end{tabular}

$D_{0}$ and $\mathrm{D}_{0}^{\prime}$ : actual and computed object sizes. $L_{\mathrm{ff}}=151 \mathrm{~mm}, F_{1}=35 \mathrm{~mm}, \Delta D_{0}=D_{0}-D_{0}^{\prime}$. 


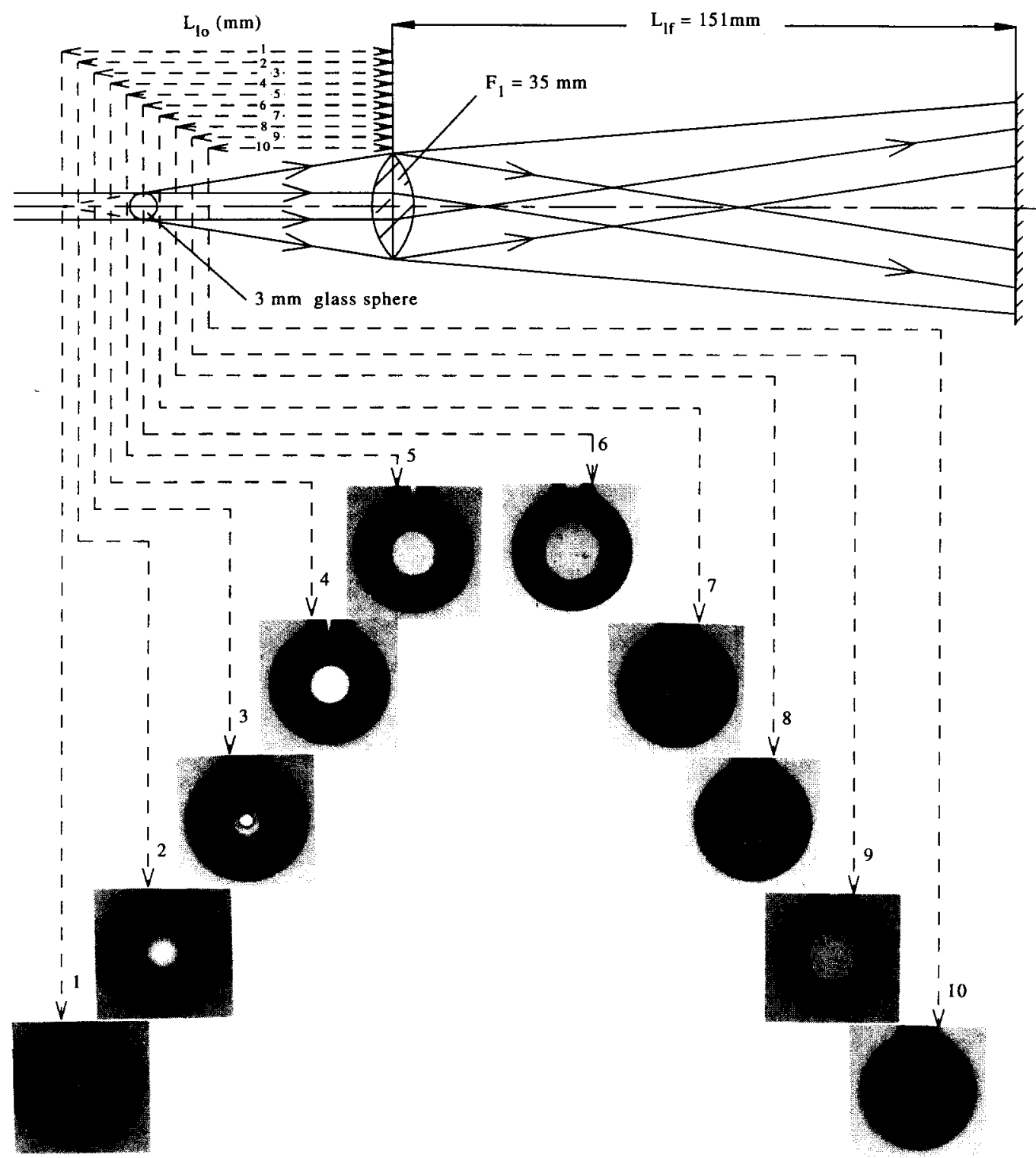

Figure 6. Photographs for validating the computation of bubble longitudinal positions (object: $3 \mathrm{~mm}$ glass spheres).

In pool boiling, it has been well established that bubble departure is relatively independent of heat flux but strongly dependent on cavity size and system pressure. As system pressure increases, bubble departure size decreases. For a smooth heating plate, an empirical correlation given by Hsu \& Graham (1976) written as:

$$
d_{\mathrm{d}}=1.614(1 / \sqrt{ } P)
$$

where $d_{\mathrm{d}}$ denotes the bubble departure size [mm] and $P$ the system pressure [bar]. In the graphic form, the relation of $d_{\mathrm{d}}-P$ is shown in figure 9.

Table 2. Comparison of computed with actual object sizes (glass spheres)

\begin{tabular}{lcccccccccc}
\hline Index & 1 & 2 & 3 & 4 & 5 & 6 & 7 & 8 & 9 & 10 \\
\hline$L_{10}(\mathrm{~mm})$ & 57.60 & 54.60 & 51.60 & 48.60 & 47.10 & 45.60 & 44.10 & 42.60 & 39.60 & 36.60 \\
$L_{\mathrm{lo}}^{\prime}(\mathrm{mm})$ & 59.11 & 55.83 & 53.07 & 48.64 & 47.15 & 45.36 & 44.10 & 42.83 & 41.10 & 41.10 \\
$\left|\Delta L_{\mathrm{lo}}\right| / L_{\mathrm{lo}}$ & $2.6 \%$ & $2.3 \%$ & $2.8 \%$ & $0.1 \%$ & $0.1 \%$ & $0.5 \%$ & $0.0 \%$ & $0.5 \%$ & $D_{\mathrm{b}}>D_{\mathrm{s}}$ & $D_{\mathrm{b}}>D_{\mathrm{s}}$ \\
\hline
\end{tabular}

$L_{\mathrm{lo}}$ and $L_{\mathrm{lo}}^{\prime}$ : actual and computed longitudinal positions.

$D_{0}=3 \mathrm{~mm}, L_{\mathrm{lf}}=151 \mathrm{~mm}, F_{1}=35 \mathrm{~mm}, \Delta L_{\mathrm{lo}}=L_{\mathrm{lo}}^{\prime}-L_{\mathrm{lo}}$. 

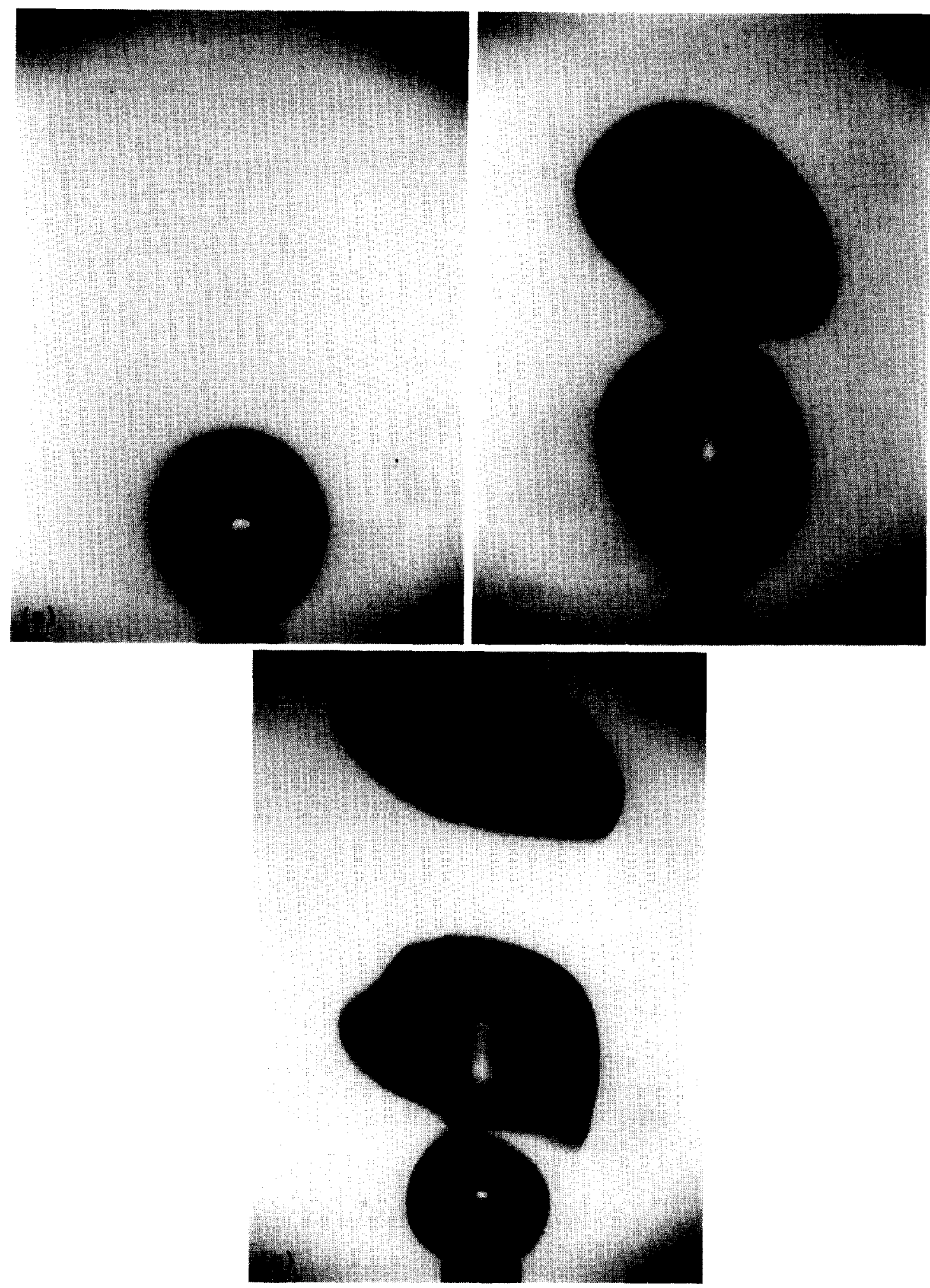

Figure 7. Photographs for validating computations of bubble longitudinal positions (object: air bubbles at mouth of injector).

For a saturated forced convection boiling flow, a bubble hardly changes shape after departure from the heating surface. This is now illustrated with the aid of two typical photographic results, taken respectively at 30 and 70 bar and shown in figure 10 . 
Table 3. Comparison of computations with actual object positions (air bubbles)

\begin{tabular}{lcccccccccc}
\hline Index & 1 & 2 & 3 & 4 & 5 & 6 & 7 & 8 & 9 \\
\hline$L_{\text {lo }}(\mathrm{mm})$ & 50.60 & 49.60 & 47.60 & 45.60 & 44.60 & 43.60 & 41.60 & 39.60 & 37.60 & 35.60 \\
$L_{\mathrm{l}}^{\prime}(\mathrm{mm})$ & 50.52 & 50.79 & 46.64 & 44.95 & 42.51 & 42.01 & 40.26 & 39.88 & 36.61 & 34.64 \\
$\left|\Delta L_{\mathrm{lo}}\right| / L_{\mathrm{lo}}$ & $0.2 \%$ & $2.4 \%$ & $2.2 \%$ & $1.4 \%$ & $4.7 \%$ & $3.6 \%$ & $3.2 \%$ & $0.1 \%$ & $2.6 \%$ & $2.7 \%$ \\
\hline
\end{tabular}

$L_{\mathrm{lo}}$ and $L_{\mathrm{lo}}^{\prime}:$ actual and computed longitudinal positions.

$L_{\mathrm{lf}}=151 \mathrm{~mm}, F_{1}=35 \mathrm{~mm}, \Delta L_{\mathrm{lo}}=L_{\mathrm{lo}}^{\prime}-L_{\mathrm{lo}}$.

According to [6], $d_{\mathrm{d}}$ equals $0.29 \mathrm{~mm}$ at $P=30$ bar and $d_{\mathrm{d}}$ equals $0.19 \mathrm{~mm}$ at $P=70$ bar. The results of figure 10 are rather close to the calculations. On the photos in figure 10, "bright spots" in some bubble images are clearly shown. Longitudinal positions of the bubbles are calculated by [4]. Positions of bubble are about $20 \mathrm{~mm}$ from the window, indicating a position close to the tube axis. More measurements are taken with the aid of a stronger light source.

\section{CONCLUDING REMARKS}

An optical system is presented in this paper to obtain shadow-photographs of bubbles in a high-pressure evaporator tube. With this system and related interpretation principles, not only the size and the velocity of a bubble can be computed from its shadow images on one photograph, but also the longitudinal position of the bubble on the optical axis may be estimated from a "bright spot" in its shadow images with a simple relation based on geometrical optics. In particular, the relation for estimating bubble longitudinal positions is, to the authors' knowledge, to be introduced for the first time and provides extra bubble information from a usual shadow photograph.

The performance of the new system and the validity of the interpretation principles were examined through three series of calibration tests, using a calibration test setup to simulate situations in the high pressure tube. Very satisfactory results were provided. In the first and second series, relative deviations of estimations of sizes and longitudinal positions of transparent objects
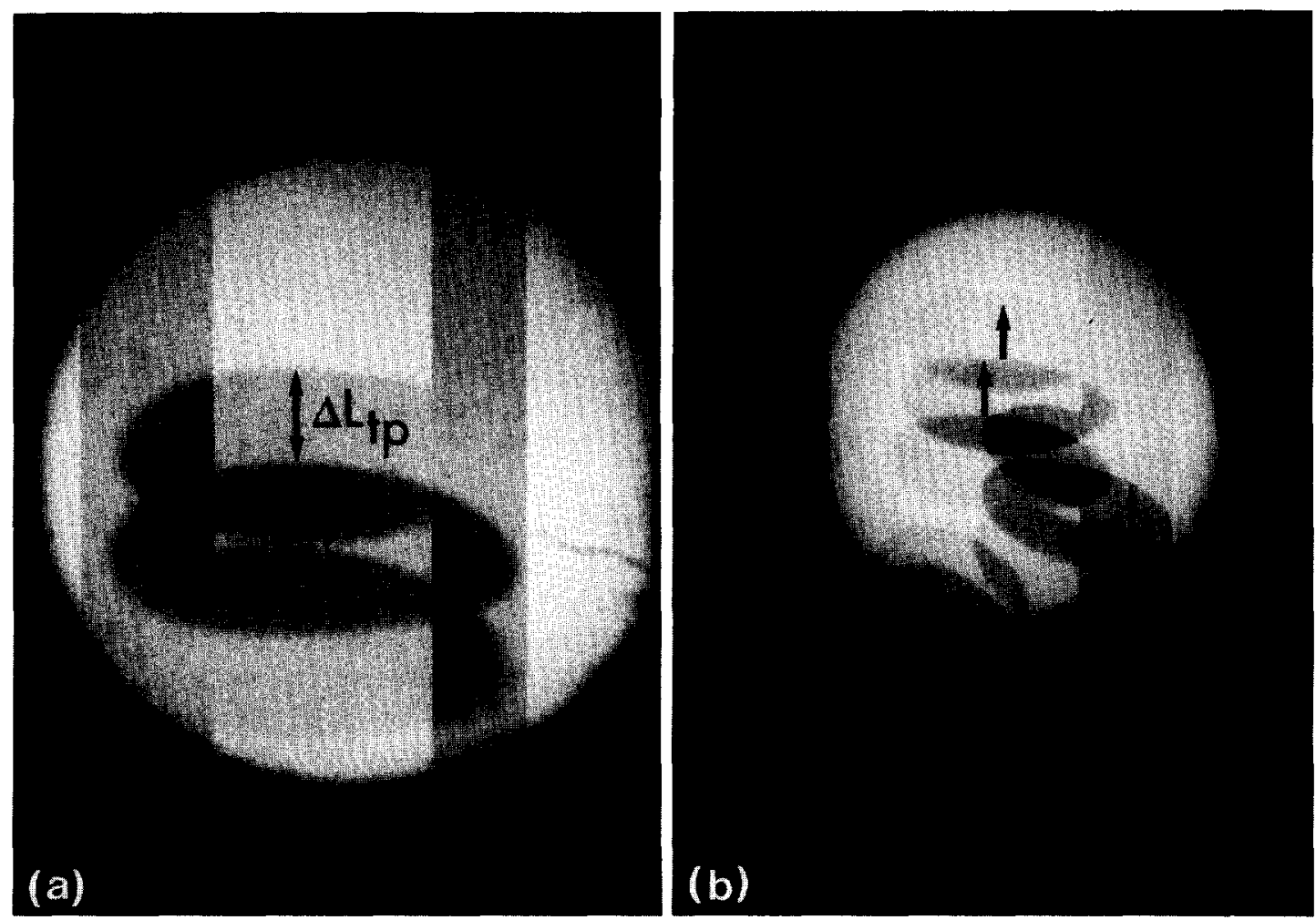

Figure 8. Two photographs of calibration tests for determining bubble velocities. Case a: $F R Q_{n}=375 \mathrm{~Hz}$, $\Delta L_{2 \mathrm{p}}=1.8 \mathrm{~mm}, \rightarrow \mathrm{VEL}_{\mathrm{b}}=0.68 \mathrm{~m} / \mathrm{s} ;$ Case b: $\mathrm{FRQ}_{\mathrm{n}}=375 \mathrm{~Hz}, \Delta L_{2 \mathrm{p}}=1.2 \mathrm{~mm}, \rightarrow \mathrm{VEL}_{\mathrm{b}}=0.45 \mathrm{~m} / \mathrm{s}$. 


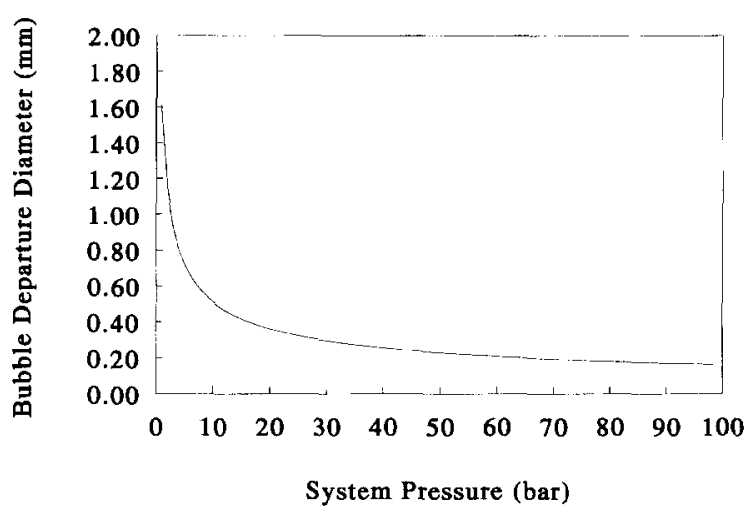

Figure 9. Vapour bubble departure diameter versus system pressure.

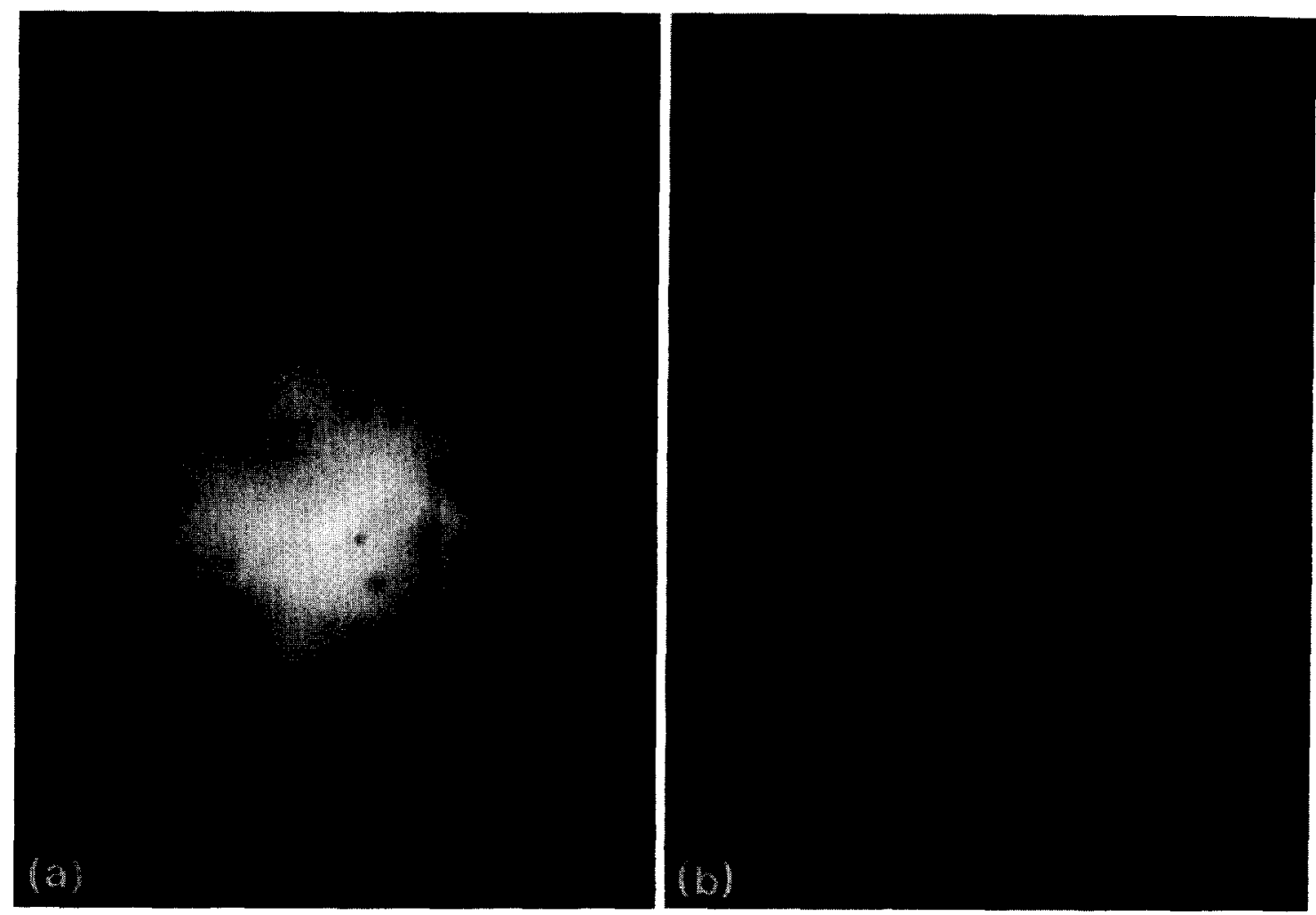

Figure 10. Photographs of bubbles in a high pressure evaporator tube. Tube diameter $39 \mathrm{~mm}$; mean water flow velocity in the tube $1.5 \mathrm{~m} / \mathrm{s}$; Case a: $P=30$ bar; Case b: $P=70$ bar.

to installations are respectively within 1 and $5 \%$. With the aid of a nanolight, velocities of bubbles were measured.

The optical system was successfully applied in a high-pressure evaporator tube. Experimental results of bubble sizes are close to the computations from empirical correlations. "Bright spots" were obtained at 30 and 70 bar. More accurate and clear results are expected with the aid of a stronger light source.

Because of the generality of the system developed, the principle to determine the longitudinal positions of a bubble from the size of a bright spot may be extended to other dispersed two-phase flow situations.

Acknowledgements - The authors would like to acknowledge Mr T. Wijchers of Prins Maurits Laboratory (TNO, The Netherlands) for his valuable suggestions and Mr J. Verspagen of Eindhoven University of Technology for his assistance in preparing the figures. 


\section{REFERENCES}

Cheremisinoff, N. P. 1986 Measurement techniques for multiphase flows, (Gas-Liquid flows). Encyclopedia of Fluid Mechanics, Vol. 2, pp. 1281-1338.

HewIt, G. F. 1978 Measurements of Two-phase Flow Parameters, pp. 105-173. Academic Press. Hsu, Y. \& Graham, R. M. 1976 Transport Processes in Boiling and Two-phase Systems, pp. 1-45. Hemisphere Publishing Corporation.

JONES, O. C. 1983 Two-phase flow measurement techniques in gas-liquid systems. In Fluid Mechanics Measurements (Edited by GoldSTEIN, R. J.), pp. 479-558. Springer-Verlag.

KLEIN, M. V. 1986 Optics, Second Edition, pp. 129-257. Wiley.

Meng, H., Moonen, G., Boot, P. \& VAN Der Geld, C. W. M. 1992 Phase distribution measurements around obstacles in a vertical tube. Int. Symp. on In-situ Measuring Techniques in Multiphase Flows (ASME publication 92-WA/FE-2), ASME Winter Annual Meeting, Anaheim, U.S.A. 8-13 November 1992, pp. 1-8.

MeNG, H. 1993 On disperse two-phase flows past obstacles. Ph.D thesis, Eindhoven University of Technology, pp. 26-67.

Morton, R. A. 1984 Photography for the Scientist, Second Edition, pp. 87-166. Academic Press.

VAN DER GeLD, C. W. M. 1983 A flexible optical measuring device for special environments, Technical Report WPS3-83.06.R354, Eindhoven University of Technology, pp. 1-50.

VAN DER GeLD, C. W. M. 1985 On the phase distribution transitions in vertical evaporator tubes. Ph.D thesis, Eindhoven University of Technology, pp. 36 40.

Van Stralen, S. \& Graham, R. M. 1979 Boiling Phenomena, pp. 329-362. Hemisphere Publishing Corporation. 